\title{
All-Trans Retinoic Acid, A Derivative of Vitamin A, Improved Intestinal Epithelial Barrier Function Through PFKP
}

\section{Yongwu Xie}

Zhuhai Center for Maternal and Health Care

\section{Shuyu Huang}

Zhuhai Center for Maternal and Child Health Care

Ting Liu

Zhuhai Center for Maternal and Child Health Care

Huiyan Sun

Zhuhai Center for Maternal and Child Health Care

Haoyan Ren

Zhuhai Center for Maternal and Child Health Care

Chunqi Xie ( $\nabla$ xcqeyk@163.com )

Zhuhai Center for Maternal and Health Care https://orcid.org/0000-0002-8663-4682

\section{Research}

Keywords: All-trans Retinoic Acid, Muc2, Differentiation, PFKP, Intestinal Barrier Function

Posted Date: August 10th, 2020

DOl: https://doi.org/10.21203/rs.3.rs-55382/v1

License: (c) (i) This work is licensed under a Creative Commons Attribution 4.0 International License.

Read Full License 


\section{All-trans Retinoic Acid, A Derivative of Vitamin A, Improved Intestinal}

\section{Epithelial Barrier Function through PFKP}

3 Yongwu Xie ${ }^{1, *}$, Shuyu Huang ${ }^{1, *}$, Ting Liu ${ }^{2}$, Huiyan Sun ${ }^{1}$, Haoyan Ren ${ }^{1, \#}$, Chunqi Xie ${ }^{1, \#}$

4 1. Department of Pediatrics, Zhuhai Center for Maternal and Child Health Care, Zhuhai, 5 China;

6 2. Department of Rheumatology, Zhuhai Center for Maternal and Child Health Care, Zhuhai,

7 China;

$8 \quad$ \# These authors contributed equally to this work.

$9 \quad *$ These authors are co-responding authors.

10 Running title: the function of ATRA in differentiation

11 Address correspondence to:

12 Department of Pediatrics

13 Zhuhai Center for Maternal and Child Health Care,

14 Zhuhai, 519000, China;

15 Email: xcqeyk@163.com (Chunqi Xie)

$16995410302 @ q q . c o m$ (Haoyan Ren);

17 


\section{Abstract}

19 Objective: Mucosal Healing, relied on the coordinated activity of IECs for improvement of 20 intestinal barrier function, is the critical goal in treatment of IBD. All-trans retinoic acid 21 (ATRA) is known to regulates cell proliferation and differentiation. The aims of the present 22 study were to investigate the effects of ATRA on the intestinal differentiation.

23 Methods: We collected the clinical sample from the patients to analyze the vitamin A, 24 TEER, western blotting and real-time PCR were performed to detect the effect of vitamin 25 A on PFKP expression and intestinal epithelial cell differentiation.

26 Results: In this study, we showed that increased TEER and decrease paracellular permeability of IECs were induced by ATRA in dose-dependent manner, which is attributed to enhanced MUC2 expression, a marker of goblet cell by western blotting, real-time PCR and TEER assay, while no significant difference of villin was found to alter. The further results show that ATRA suppressed PFKP expression in IECs, while overexpression of PFKP could reverse the promotion of ATRA on MUC2 expression, implying ATRA-induced MUC2 expression in PFKP-dependent manner and in dose-dependent way. The clinical sample analysis suggested vitamin $A$ is not significant associated with gender, and replenishment of vitamin A is critical for intestinal differentiation.

Conclusion: ATRA improved intestinal epithelial differentiation via PFKP-mediated Muc2 expression. The findings suggest that ATRA could serve as a novel therapeutic agent to ameliorate development of inflammatory bowel disease. 


\section{Introduction}

Metabolic reprograming, involved in a various of disease, in the niche is critical for intestinal stem cell function. The studies have showed that energy metabolism regulates self-renewal and differentiation of intestinal stem cells, leading to differentiation of goblet cell characterized by the increased Mucin2(MUC2) expression, Paneth cells as shown by the induction of lysozyme (LYZ), and enterocytes (characterized by expression of the brush-border enzymes IAP and SI, villin, and Keratin 20(KRT20))(1, 2). For instance, mitochondrial oxidative phosphorylation (OXPHOS) activity drives differentiation and crypt formation by a mechanism that involves p38 activation through mitochondrial reactive oxygen species (ROS) signaling, and the glycolytic phenotype mediated by glycolytic key enzymes, including HK2, PFKP, in Paneth cells and increased OXPHOS in Lgr5' (crypt base columnar cells) $\mathrm{CBCs}$ are required in supporting both niche and stem cell function(3). In addition, Gao et al have recently showed that liver kinase B1 (Lkb1)-regulated energy metabolism not only exerts a crucial impact on cell survival, but also plays an important role in cell fate determination(4).

All-trans Retinoic Acid (ATRA), the predominant natural metabolite of vitamin A derived from animal and plant food sources, exhibited the protective effects in the intestinal mucosa, including immunomodulatory and anti diarrheal effects(5). Vitamin A deficiency has been showed to exacerbate intestinal injury in the rat model of IBD, leading to colorectal cancer progression $(6,7)$, and the patients with IBD and short bowel syndrome exhibited lower levels of vitamin $A$, indicating the critical role of vitamin $A(8,9)$. What's more, ATRA cotreatment significantly counteracted IFN- $\gamma$-induced decrease in JAK/STAT1mediated (downregulated in adenoma)DRA expression, alleviating diarrhea in gut inflammation(10), and the study also reported Vitamin $A$ is required for $T$ helper 2associated responses that are shared by immune responses to allergens and parasites(1113) and generally inhibits the development of M1 macrophage and THP-1 cells, leading to reduce TNF, CCL3 and CCL4 level and modulate tissue remodeling $(14,15)$. In addition to macrophages, Whether the involvement of IECs in ATRA-induced mucosal healing is unknown in IBD. 
The intestinal epithelium is a rapidly renewing tissue which plays central roles in nutrient absorption, barrier function, defense against harmful microorganisms and lumen content and the prevention of intestinal inflammation(16). Goblet cells is the primary player in maintaining intestinal mucosal barrier, which is attributed to the mucus layers secreted by goblet cells and contains commensal bacteria, constitutes the first line of defense against pathogenic gut microbiota(17). The homeostasis of intestinal barrier is based on a delicate regulation of epithelial metabolism and differentiation, which is closely association with intestinal development $(1,2)$. impairment in intestinal epithelial integrity and barrier function is associated with many diseases, which is a etiological factors of inflammatory bowel disease (IBD) and other digestive tract diseases $(18,19)$. In this study, the results from population investigation showed that the $88.4 \%$ of 2930 subjects have the normal level of vitamin A level, much more attenuation is required to improve those who lacks enough vitamin A. most importantly, we found that ATRA promoted intestinal epithelial barrier function by inducing MUC2, not villin, expression in IECs, leading to increase intestinal barrier function and decrease paracellular permeability, which is attributed to attenuation of PFKP expression caused by ATRA stimulation, while overexpression of PFKP in HT-29 cells overcame the effect of ATRA on MUC2 expression

\section{Materials and Methods}

Reagents and antibody

90 Vitamin A (R2625) and DMSO(D2650) were obtained from Sigma (St. Louis, MO, USA).

91 Culture medium and fetal bovine serum were purchased from life technologies. Trizol was from invitrogen (Invitrogen, Thermo Fisher Scientific). All-in-one ${ }^{\mathrm{TM}}$ first-strand cDNA synthesis kit and All-in-one ${ }^{\mathrm{TM}}$ qPCR mix were from Genecopoeia ${ }^{\mathrm{TM}}$ (FulenGen, Guangzhou,

94 China). Pierce BCA Protein Assay Kit were purchased from thermo fisher scientific, Villin (ab201989), MUC2(ab272692) and PFKP(ab204131) were purchased from Abcam

96 (Cambridge, UK). alpha-tubulin(AC013) were purchased from Abclonal (Wuhan, China).

97 Other reagents used in this study were purchased from Sigma(St.Louis, MO, USA). 
CaCO2 and HT-29 cells were obtained from American Type Cell Collection(Manassas, VA),

100 cells were cultured in DMEM supplemented with $10 \%$ FBS. The cells were maintained at $37^{\circ} \mathrm{C}$ in a humidified $5 \% \mathrm{CO}_{2}$ incubator. Cells were stimulated with vitamin $\mathrm{A}$ as described in the study(5), ATRA were dissolved in DMSO and stored at $-80^{\circ} \mathrm{C}$. plasmids were purchased from addgene and transfected into cells with Hilymax (H357, Dojindo, Japan) following the manufacturer's instructions.

\section{RNA extraction and quantitative real-time PCR}

Total RNA was collected and converted to CDNA using the All-in-one ${ }^{\mathrm{TM}}$ first-strand cDNA synthesis kit and amplified by PCR using the All-in-one ${ }^{T M}$ qPCR mix according to the manufacturer's instructions. Primer sequences for PFKP and MUC2 were listed as followed:

\begin{tabular}{|c|c|c|}
\hline Genes & Forwards $\left(3^{\prime}-5^{\prime}\right)$ & Reverse $\left(3^{\prime}-5^{\prime}\right)$ \\
\hline PFKP & GGAGATTTCAAGATGCGGTT & GCAATTGGTCTTTGGGATCT \\
\hline MUC2 & GAAGGGAGTGACCATCATCG & TTAAAGCACCCAGGCTTGAT \\
\hline GAPDH & TCACCACCATGGAGAAGGC & GCTAAGCAGTTGGTGGTGCA \\
\hline
\end{tabular}

109

western blotting

111 As described in study(20), the whole protein were collected in denaturing SDS sample buffer and analyzed by western blotting, and transferred to a $0.22-\mu \mathrm{m}$ nitrocellulose

113 transfer membrane. The membrane was blocked with 5\% (w/v) milk in PBS/0.05\% (v/v)

114 Tween-20 and incubated with the indicated antibody overnight at $4^{\circ} \mathrm{C}$ followed by incubation with a horseradish peroxidase secondary antibody (Jackson ImmunoResearch)

116 for $1 \mathrm{~h}$ at room temperature. Proteins were detected using an enhanced 117 chemiluminescence (Perkin Elmer).

\section{Vitamin A level measurement}

119 The serum was collected from hospitalized patients or clinical lab, which were measured

120 by AB SCIEX Triple QuandTM 4500MD according the manufacturer's instruction.

\section{In vitro barrier function assessment.}

122 As described in Li et al study(21), briefly, CaCO2 cells were seeded in the apical chamber 123 that bathed in the basal chamber with $1.0 \mathrm{ml}$ DMEM complete medium for 21 days. Voltage 
124 was measured daily using EVOM (WPI, Sarasota, FL, USA), which was multiplied by the 125 area of filter $\left(1.12 \mathrm{~cm}^{2}\right)$ to obtain the TEER in $\mathrm{Ohm} \mathrm{cm}^{2}$. DMEM complete medium in apical 126 and basal chamber was refreshed every day. The permeability of rhodamine-dextran 127 (Sigma) across the $\mathrm{CaCO} 2$ cell monolayer was measured as previously described with 128 modifications. At 21 days, $1.0 \mathrm{mg} / \mathrm{ml}$ rhodamine -dextran was added on the apical side of 129 monolayers after washed twice with PBS. One milliliter cells in the basal chamber were 130 taken at indicated point and $1.0 \mathrm{ml}$ pre-warmed fresh medium was added after each 131 sampling to replenish basal medium. The fluorescence emission at $520 \mathrm{~nm}$ was measured 132 with excitation at $490 \mathrm{~nm}$ using Synergy $\mathrm{H} 1$ microplate reader.

\section{Statistical analysis}

134 All analysis was conducted using GraphPad Prism V software. A P value $<0.05$ was 135 considered statistically significant. Statistical differences among groups were determined 136 by Student's t-test and ANOVA in analysis mRNA or protein level.

\section{Results}

138 ATRA enhances intestinal barrier function through promoting intestinal epithelial differentiation

140 To determine whether ATRA has a role in intestinal epithelial differentiation, we used 141 ATRA to incubate with Caco2 and HT-29 of intestinal epithelial cells. As shown in Fig.1A, 142 Caco2 cells were treated with 2uM and 4uM ATRA, respectively. Treatment with ATRA in $143 \mathrm{CaCO} 2$ cell led to a significant improvement in the epithelial barrier function as shown by 144 increased transepithelial electrical resistance (TEER) during a time course mirroring that 145 the normal period required to a complete a cycle of epithelial renewal, compared the 146 control group(OuM). In line with this, paracellular permeability of intestinal barrier function 147 were also measured by addition of rhodamine-dextran. The analysis of paracellular 148 permeability of rhodamine-dextran were decreased in various of time point (Fig.1B). 149 Interestingly, both changes of TEER and paracellular permeability were in dose-dependent 150 manner.

151 ATRA contributes to induction of goblet cell marker muc2 expression 
The mucus layer in the intestine is mainly composed of mucin glycoproteins that are secreted from intestinal goblet cells. The mucus layer acts as an intestinal barrier and plays a critical role in preventing toxic molecules and pathogens from penetrating into the intestinal mucosae, thus preventing intestinal inflammation(22). Destruction of the mucus layer, secretion by intestinal goblet cells(23), causes severe intractable inflammatory bowel diseases (IBDs), including Crohn's disease and ulcerative colitis(24).

We have further showed that treatment of HT-29 and $\mathrm{CaCO} 2$ cells with ATRA at 2uM and 4uM lead to increase the mRNA expression level of MUC2 (Fig.2A). consisted with this, the protein level of MUC2 were also drastically enhanced in response to ATRA stimulation, and statistic difference were quantified. However, further analysis showed that ATRA treatment of IECs failed to alter villin expression, a differentiation marker of the differentiation of enterocytes(25), indicating that goblet cells is a response cells in response to ATRA stimulation. Taken together, these results suggested that ATRA is an inducer of intestinal cell differentiation of goblet cells.

\section{ATRA induced MUC2 expression in PFKP-dependent way}

Previously, the study showed that HMGCS2-mediated Ketogenesis contributes to intestinal cell differentiation(1), FASN has been reported to modulate intestinal barrier function through palmitoylation of MUC2(26), these finding suggested that metabolism reprogramming is a critical event in regulating differentiation Interestingly, in this our results showed that ATRA treatment in $\mathrm{HT}-29$ and $\mathrm{CaCO} 2$ cells led to a significant decrease PFKP mRNA level in dose-dependent manner (Fig.3A), in line with this, the results from western blotting and quantified results also confirmed the protein level of PFKP was reduced in $\mathrm{HT}-29$ and $\mathrm{CaCO} 2$ cells treated with ATRA(Fig.3B-C). To further determine whether PFKP, a limit key enzyme involved in glycolysis, involved in ATRA-induced intestinal cell differentiation. We tried to detect MUC2 expression in HT-29 cells treated with 2uM ATRA following by overexpression of PFKP. The results showed that ectopic expression of FKPK in HT-29 cells significantly reversed the promoting effect of ATRA on MUC2 expression (Fig.3D). Taken together, these findings suggested that ATRA-induced MUC2 expression in PFKP manner. 


\section{The characteristics of serum vitamin A level in population investigation}

183 The above results showed that ATRA induced intestinal epithelial cell differentiation in PFKP-dependent way, implying the critical role of ATRA in intestinal development. To

185 further analysis the level of vitamin A in a total of 3315 subjects collected from Zhuhai

186 Center for Maternal and Child Health Care and detailed information listed in the 187 supplementary Materials, we found that $2930(88.4 \%)$ cases was in the normal level 188 of vitamin A, while $341(10.3 \%)$ and $44(1.3 \%)$ were in the low and high level of vitamin 189 A, respectively(Fig.4A), and there is no difference between female and male in serum vitamin A(Fig.4B). Further analysis showed that no significant difference between female and male in those who are low level of serum vitamin A.

\section{Discussion}

193 Up to now, no direct available Reports on the function of ATRA in intestinal epithelial cells differentiation. In this study, we further demonstrated a novel role of ATRA in cell differentiation by regulating PFKP expression. we, for the first time, showed that ATRA induced MUC2 expression, leading to improve intestinal barrier function characterized by increased TEER, which attributed to decrease of PFKP expression. Thus, our results provided novel insight to support an important role of ATRA as a promising assistant to improve barrier function.

All-trans retinoic acid, which binds to RARa, RAR $\beta$ and RARY as a pan RAR agonist, has shown to promote differentiation of various endodermal tissues, including lung, pancreas and bladder and tissues consisting of squamous cells, such as skin and cornea $[11,12]$, in several studies using human cells. In mouse models, retinoid acid signaling enhanced the terminal differentiation and proliferation of esophageal progenitor cells $[13,14]$. What's more, the levels of inflammatory factors were significantly reduced, while the expression levels of claudin-1, occludin, and ZO-1 were increased, leading to increase intestinal barrier function after the VA and RA treatments. Meanwhile, TEER was increased 
209 treatment both in vivo and in vitro(27). In addition, our results further showed that ATRA-

210 induced barrier function by inducing MUC2, not villin, expression. interestingly, goblet cells

211 is characterized by increased MUC2 expression in IECs(28), implying that ATRA primary

212 contributed goblet cells differentiation to improve intestinal barrier function.

213 mTORC1 signaling is well known to be a central regulator of intestinal function.

214 Inhibition of mTORC1 activity/expression could promote HMGCS2-induced CDX2 215 expression, leading to increase intestinal epithelial function(1), and tuberous sclerosis 2

216 (TSC2) positively regulates the expression of the goblet cell differentiation marker, MUC2,

217 in intestinal cells(29). Interestingly, ATRA dose-dependently reduced the phosphorylation

218 levels of mTOR and mTOR target proteins p70 S6 kinase (p70S6K) and 4E-binding protein

2191 (4EBP1) in A7r5 and HASMC(30), leading to increase CDX2 expression and decrease 220 PFKP expression during intestinal epithelial cell differentiation(1). However, in addition to 221 mTORC1 signaling, the further work is required to address the mechanism by which ATRA-

222 mediated PFKP expression, and whether retinoic acid receptor (RAR), including RARa,

223 RAR $\beta$ and/or RARY, involved in PFKP expression in response to ATRA stimulation.

224 The function of ATRA in intestinal function is gradually becoming more and more 225 important, based on the present findings, a total of 3315 subjects was enrolled in our 226 study to analyze the level of vitamin A, the detailed information were listed in 227 Supplementary Materials, the results showed that $2930(88.4 \%)$ subjects were in the normal level of vitamin $\mathrm{A}(0.3-0.7)$, and $44(1.3 \%)$ were higher than normal. While there are still $340(10.3 \%)$ cases were found under normal level. These findings suggested that alterations in vitamin A level is critical to induce different biological function in intestine development. Further studies are warranted to elucidate the role of vitamin A-mediated metabolism network in intestinal epithelial cells.

\section{Conclusion}

234 Collectively, the results demonstrated the critical function of ATRA, a metabolite of 235 vitamin $A$, in intestinal epithelial cell differentiation. replenishment of vitamin $A$ is an 236 effective strategy to improve intestinal epithelial barrier function.

\section{Abbreviations}


4EBP 1: 4E-binding protein 1; ATRA: All-trans Retinoic Acid; CBCs: crypt base columnar cells; CDX2: Caudal type homeobox 2; HK2: hexokinase 2; IECs: intestinal epithelial cells; IAP: Intestinal Alkaline phosphatase activity; IBD: inflammatory bowel disease; KRT20: Keratin 20; LKB1:liver kinase B1; LYZ: lysozyme; MPC: mitochondrial pyruvate

242 carrier; MUC2: Munic2; mTORC1: mammalian target of rapamycin complex 1; OXPHOS: oxidative phosphorylation; PFKP: Phosphofructokinase, platelet; p70S6K: p70 S6 kinase; RAR: retinoic acid receptor ROS: reactive oxygen species; SI: sucrase-isomaltase; TSC: tuberous sclerosis 2;

\section{Declarations}

\section{Ethics approval and consent to participate}

248 A total of 3914 were enrolled in this study upon the declaration of Helsinki as reflected in 249 a prior approval approved by Medical Ethics Committee for Clinical Ethical Review of Zhuhai Center for Maternal and Child Health Care. Informed consent was given by the caregiver of the child for clinical records used, which are not publicly available, however,

252 it could be available upon reasonable request.

\section{Consent for publication}

254 Not applicable

\section{Availability of data and material}

256 The datasets generated during and/or analyses during the current study are available from the corresponding author on reasonable request.

\section{Competing interests}

259 The authors declare that they have no competing interests

\section{Funding}

261 This research did not receive any specific grant from funding agencies in the public, 262 commercial, or not-for-profit sectors.

\section{Author contributions}

264 CQX and HYR conceived and designed the experiments, YWX, SYH, TL, HYS and HYR 265 performed experiments and analyzed data, YWX and CQX wrote the manuscript, and all authors read and approved the final manuscript. 
268 We would like to thank doctors in department of Pediatrics for kindly supporting.

269

270

271

272

273

274

275

276

277

278

279

280

281

282

283

284

285

286

287

288

289

290

291

292

293

294

295

296

297

298

299

300

301

302

303

304

305

306

307

308

309

\section{Supplementary material}

The detailed information of patients enrolled in the study.

\section{Reference:}

1. Wang $Q$, Zhou $Y$, Rychahou P, Fan TW, Lane AN, Weiss HL, et al. Ketogenesis contributes to intestinal cell differentiation. Cell Death Differ. 2017;24(3):458-68.

2. Sun $X$, Yang $Q$, Rogers CJ, Du M, Zhu MJ. AMPK improves gut epithelial differentiation and barrier function via regulating Cdx2 expression. Cell Death Differ. 2017;24(5):819-31.

3. Rodriguez-Colman MJ, Schewe M, Meerlo M, Stigter E, Gerrits J, Pras-Raves M, et al. Interplay between metabolic identities in the intestinal crypt supports stem cell function. Nature. 2017;543(7645):424-7.

4. Gao Y, Yan Y, Tripathi S, Pentinmikko N, Amaral A, Paivinen P, et al. LKB1 Represses ATOH1 via PDK4 and Energy Metabolism and Regulates Intestinal Stem Cell Fate. Gastroenterology. 2020;158(5):1389-401 e10.

5. Priyamvada S, Anbazhagan AN, Kumar A, Chatterjee I, Borthakur A, Saksena S, et al. All-trans Retinoic Acid Counteracts Diarrhea and Inhibition of Downregulated in Adenoma Expression in Gut Inflammation. Inflamm Bowel Dis. 2020;26(4):534-45.

6. Bhattacharya N, Yuan R, Prestwood TR, Penny HL, DiMaio MA, Reticker-Flynn NE, et al. Normalizing Microbiota-Induced Retinoic Acid Deficiency Stimulates Protective CD8(+) T Cell-Mediated Immunity in Colorectal Cancer. Immunity. 2016;45(3):641-55.

7. Hall JA, Cannons JL, Grainger JR, Dos Santos LM, Hand TW, Naik S, et al. Essential role for retinoic acid in the promotion of CD4(+) T cell effector responses via retinoic acid receptor alpha. Immunity. 2011;34(3):435-47.

8. Reifen R, Nur T, Ghebermeskel K, Zaiger G, Urizky R, Pines M. Vitamin A deficiency exacerbates inflammation in a rat model of colitis through activation of nuclear factor-kappaB and collagen formation. J Nutr. 2002;132(9):2743-7.

9. Warden RA, Noltorp RS, Francis JL, Dunkley PR, O'Loughlin EV. Vitamin A deficiency exacerbates methotrexate-induced jejunal injury in rats. J Nutr. 1997;127(5):770-6.

10. Saksena S, Singla A, Goyal S, Katyal S, Bansal N, Gill RK, et al. Mechanisms of transcriptional modulation of the human anion exchanger SLC26A3 gene expression by IFN\{gamma\}. Am J Physiol Gastrointest Liver Physiol. 2010;298(2):G159-66.

11. Carman JA, Pond L, Nashold F, Wassom DL, Hayes CE. Immunity to Trichinella spiralis infection in vitamin A-deficient mice. J Exp Med. 1992;175(1):111-20.

12. Cantorna MT, Nashold FE, Hayes CE. In vitamin A deficiency multiple mechanisms establish a regulatory $T$ helper cell imbalance with excess Th1 and insufficient Th2 function. J Immunol. 1994;152(4):1515-22.

13. Schuster GU, Kenyon NJ, Stephensen CB. Vitamin A deficiency decreases and high dietary vitamin $A$ increases disease severity in the mouse model of asthma. J Immunol. 2008;180(3):1834-42.

14. Wang $X$, Allen C, Ballow M. Retinoic acid enhances the production of IL-10 while reducing the synthesis of IL-12 and TNF-alpha from LPS-stimulated monocytes/macrophages. J Clin Immunol. 2007;27(2):193-200. 
15. Wojtal KA, Wolfram L, Frey-Wagner I, Lang S, Scharl M, Vavricka SR, et al. The effects of vitamin A on cells of innate immunity in vitro. Toxicol In Vitro. 2013;27(5):1525-32. 16. van der Flier LG, Clevers $H$. Stem cells, self-renewal, and differentiation in the intestinal epithelium. Annu Rev Physiol. 2009;71:241-60.

17. Cai R, Cheng C, Chen J, Xu X, Ding C, Gu B. Interactions of commensal and pathogenic microorganisms with the mucus layer in the colon. Gut Microbes. 2020;11(4):680-90.

315

18. Turner JR. Intestinal mucosal barrier function in health and disease. Nat Rev Immunol. 2009;9(11):799-809.

19. Xia Z, Huang L, Yin P, Liu F, Liu Y, Zhang Z, et al. L-Arginine alleviates heat stressinduced intestinal epithelial barrier damage by promoting expression of tight junction proteins via the AMPK pathway. Mol Biol Rep. 2019;46(6):6435-51.

20. Xu W, Zhang Z, Zou K, Cheng Y, Yang M, Chen H, et al. MiR-1 suppresses tumor cell proliferation in colorectal cancer by inhibition of Smad3-mediated tumor glycolysis. Cell Death Dis. 2017;8(5):e2761.

21. Li M, Zhao J, Cao M, Liu R, Chen G, Li S, et al. Mast cells-derived MiR-223 destroys intestinal barrier function by inhibition of CLDN8 expression in intestinal epithelial cells. Biol Res. 2020;53(1):12.

327

328

22. Schroeder BO. Fight them or feed them: how the intestinal mucus layer manages the gut microbiota. Gastroenterol Rep (Oxf). 2019;7(1):3-12.

23. Dorofeyev AE, Vasilenko IV, Rassokhina OA, Kondratiuk RB. Mucosal barrier in ulcerative colitis and Crohn's disease. Gastroenterol Res Pract. 2013;2013:431231.

24. Davidson A, Diamond B. Autoimmune diseases. N Engl J Med. 2001;345(5):340-50.

25. Wang $Q$, Wang $X$, Hernandez A, Kim S, Evers BM. Inhibition of the phosphatidylinositol 3-kinase pathway contributes to HT29 and Caco-2 intestinal cell differentiation. Gastroenterology. 2001;120(6):1381-92.

26. Wei X, Yang Z, Rey FE, Ridaura VK, Davidson NO, Gordon JI, et al. Fatty acid synthase modulates intestinal barrier function through palmitoylation of mucin 2. Cell Host Microbe. 2012;11(2):140-52.

338 27. Xiao S, Li Q, Hu K, He Y, Ai Q, Hu L, et al. Vitamin A and Retinoic Acid Exhibit Protective Effects on Necrotizing Enterocolitis by Regulating Intestinal Flora and Enhancing the Intestinal Epithelial Barrier. Arch Med Res. 2018;49(1):1-9. 28. Zhou Y, Wang Q, Guo Z, Weiss HL, Evers BM. Nuclear factor of activated T-cell c3 inhibition of mammalian target of rapamycin signaling through induction of regulated in development and DNA damage response 1 in human intestinal cells. Mol Biol Cell. 2012;23(15):2963-72.

345 29. Zhou Y, Rychahou P, Wang Q, Weiss HL, Evers BM. TSC2/mTORC1 signaling controls 346 Paneth and goblet cell differentiation in the intestinal epithelium. Cell Death Dis. $347 \quad 2015 ; 6: e 1631$.

348 30. Zhang J, Deng B, Jiang X, Cai M, Liu N, Zhang S, et al. All-Trans-Retinoic Acid Suppresses 349 Neointimal Hyperplasia and Inhibits Vascular Smooth Muscle Cell Proliferation and Migration via Activation of AMPK Signaling Pathway. Front Pharmacol. 2019;10:485. 
353 Fig.1 ATRA improved intestinal barrier function. (A) CaCO2 cells of IECs were digested and seeded in 12-transwell plate and treated with ATRA as indicated concertation for 20 days, and TEER were measured every two-days and analyzed by One-way ANOVA, $\mathrm{n}=3, * * * \mathrm{P}<0.001 ; * * \mathrm{P}<0.01$. Error bars indicated s.d. (B) Rhodamine labelled dextran paracellular intestinal epithelial permeability were performed at 28 days post incubation and were detected at various of time points. One-way ANOVA analysis, $n=3, * * p<0.01$; Error bars indicated s.d.

Fig.2 ATRA contributed MUC2 expression in IECs. (A) Real time-PCR analysis of MUC2 expression level in HT-29 and $\mathrm{CaCO} 2$ cells with or without ATRA stimulation for 48 hours, respectively. The statistical difference was performed by one-way ANOVA analysis, $\mathrm{n}=3, * * * \mathrm{P}<0.001 ; * * \mathrm{P}<0.01$. Error bars indicated s.d. (B) left panel:HT-29 and CaCO2 cells were treated with or with ATRA for 48 hours, and the total protein were collected and analyzed indicated protein level. Right panel: the quantitation of MUC2 band in the western blotting were analyzed. (C) the intensity of villin were quantified in HT-29 and $\mathrm{CaCO} 2$ cells in indicated group and analyzed, respectively.

Fig.3 ATRA induced MUC2 expression in PFKP-dependent way. (A) real-time PCR analysis of PFKP in HT-29 and $\mathrm{CaCO} 2$ cells treated with indicated concentration of ATRA. (B) western blotting was performed to detect PFKP expression in $\mathrm{HT}-29$ and $\mathrm{CaCO} 2$ cell in indicated group, and intensity band were quantified and analyzed the statistical difference (C). (D) western blotting was used to analyze MUC2 expression in indicated group in HT-29 cells, and the protein level of MUC2 were quantified and analyzed.

Fig.4 Clinical characteristic of Vitamin A. (A) the serum was collected to measure the 
379 performed based on gender; (C) statistical difference were performed to analyze the 380 population of low vitamin A that marked red box in (A) up on gender.

381 
Fig.1

A

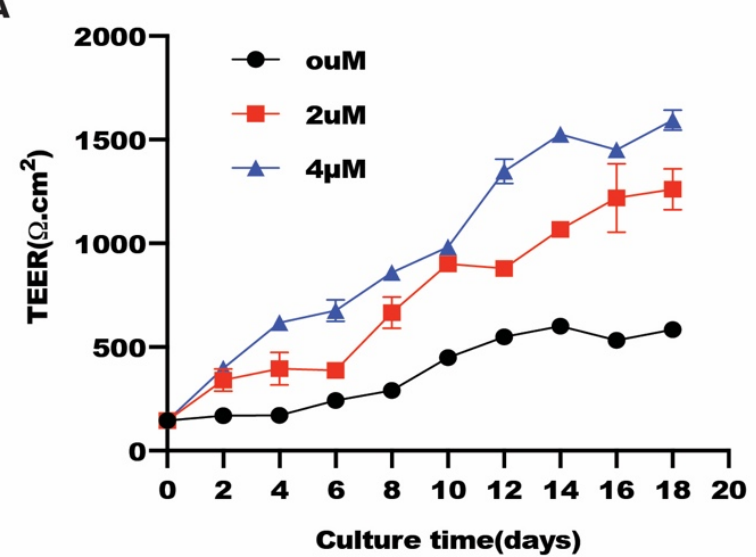

B

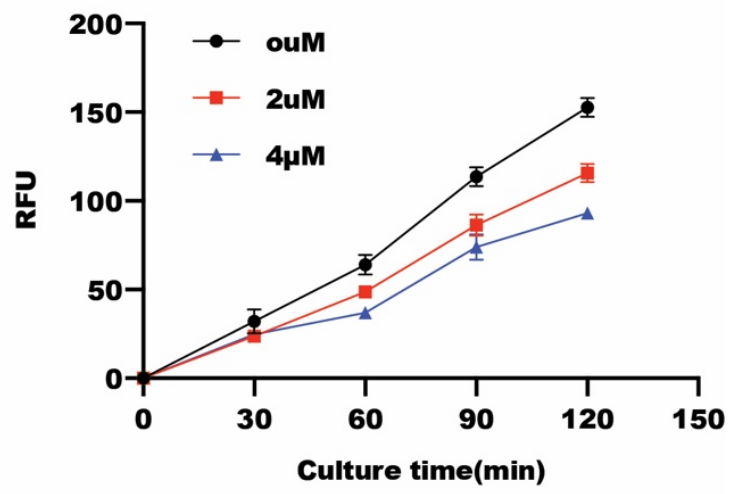

Fig.2
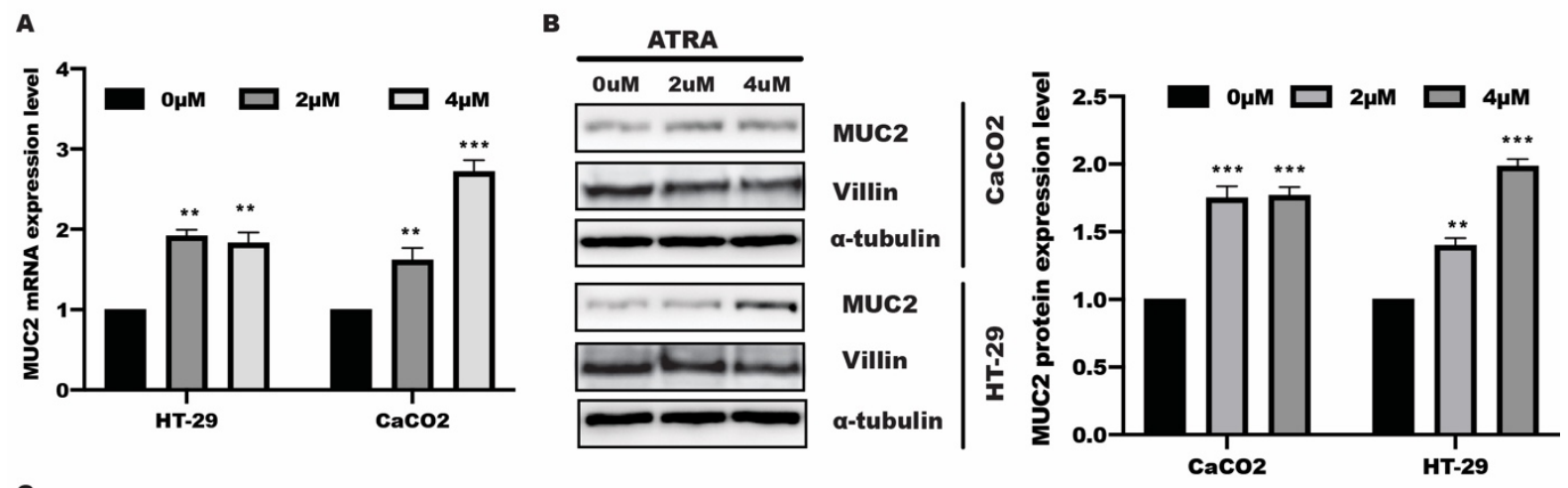

c

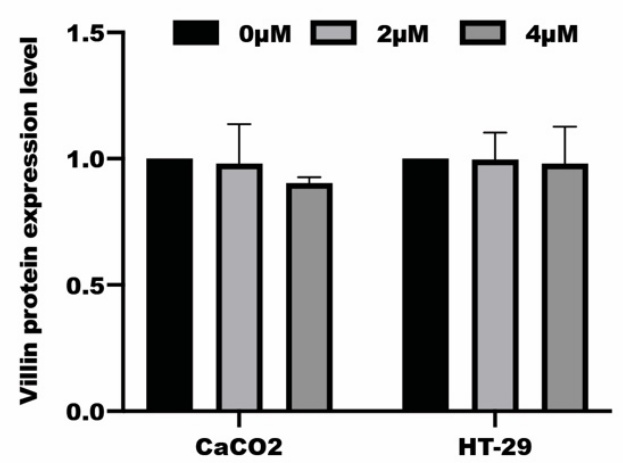


Fig.3

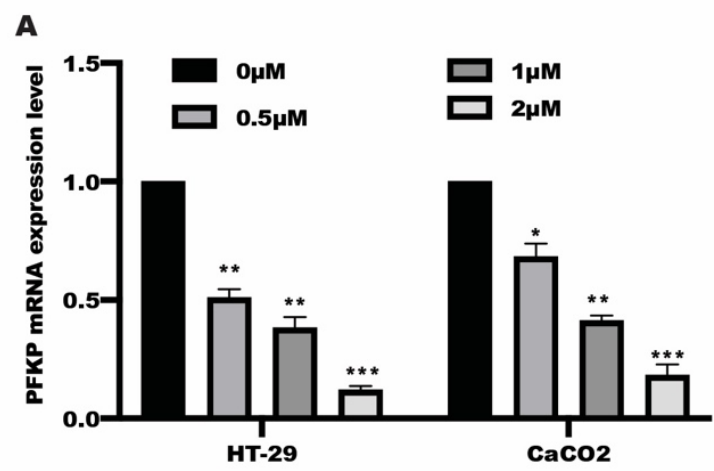

B
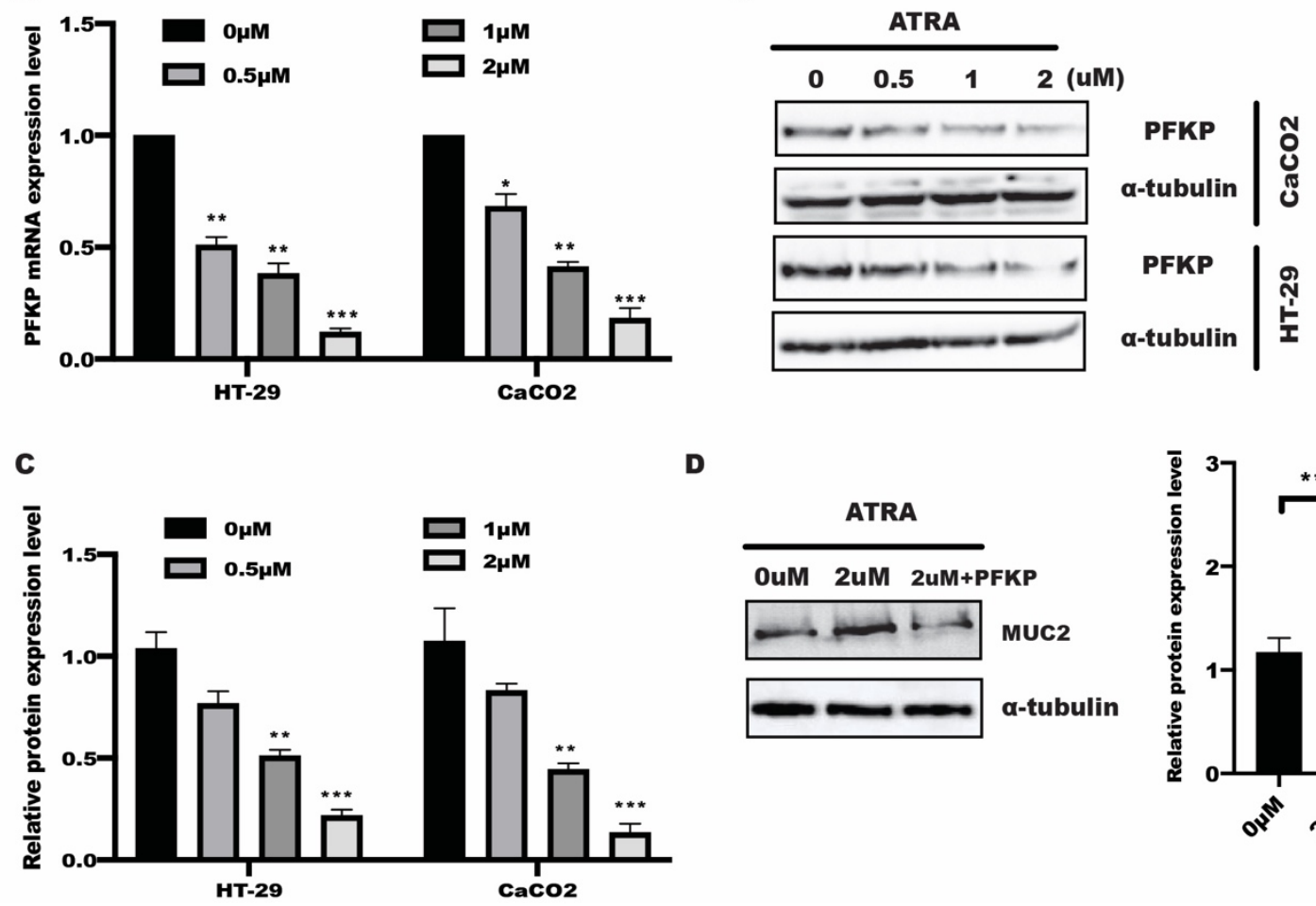

D
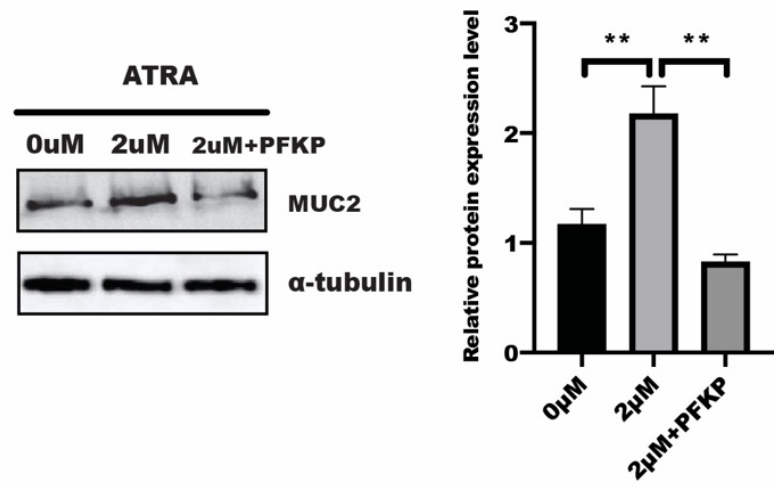

391

392

393

394

395

Fig.4

A

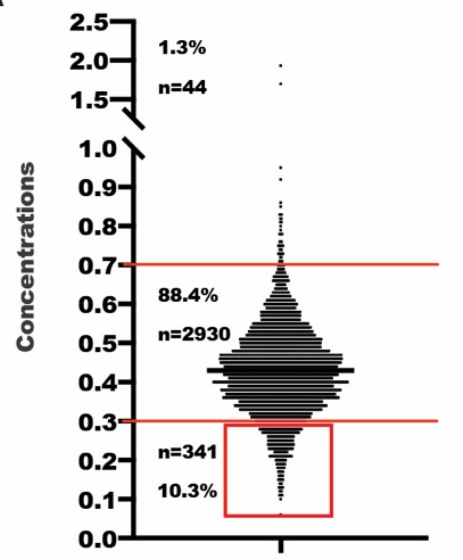

396

Vitamin A

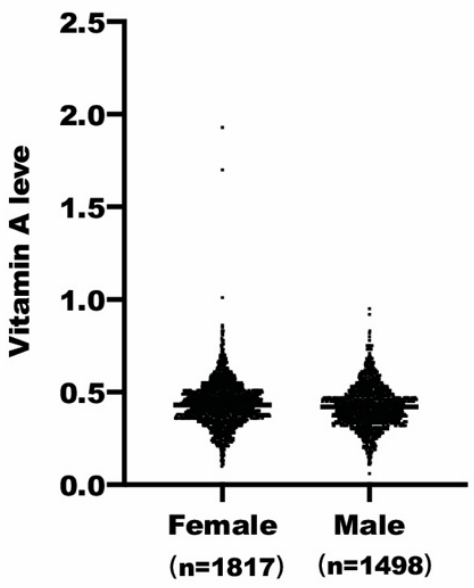

B

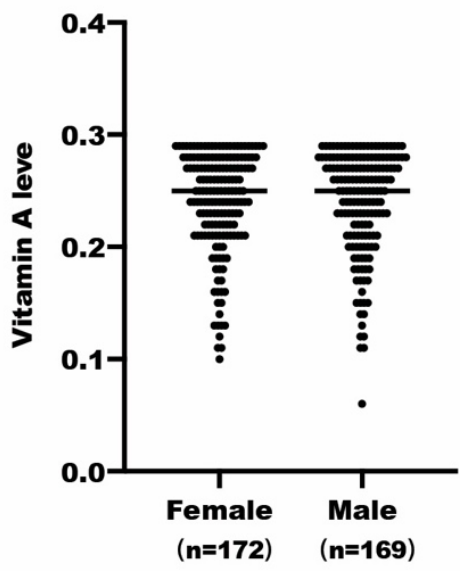


Figures

Fig.1
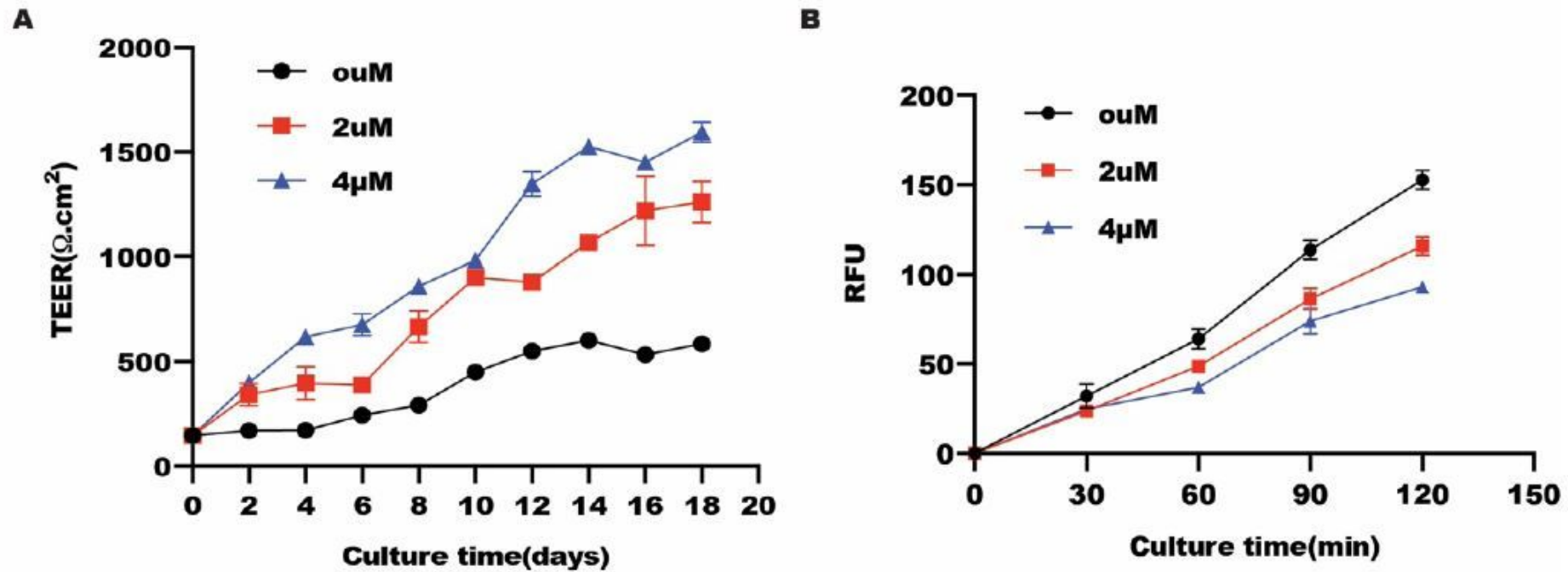

Figure 1

ATRA improved intestinal barrier function. (A) CaCO2 cells of IECs were digested and seeded in 12transwell plate and treated with ATRA as indicated concertation for 20 days, and TEER were measured every two-days and analyzed by One-way ANOVA, $n=3, * \star \star P<0.001 ; * \star P<0.01$. Error bars indicated s.d. (B) Rhodamine labelled dextran paracellular intestinal epithelial permeability were performed at 28 days post incubation and were detected at various of time points. One-way ANOVA analysis, $n=3,{ }^{* *} p<0.01$; Error bars indicated s.d. 
Fig.2

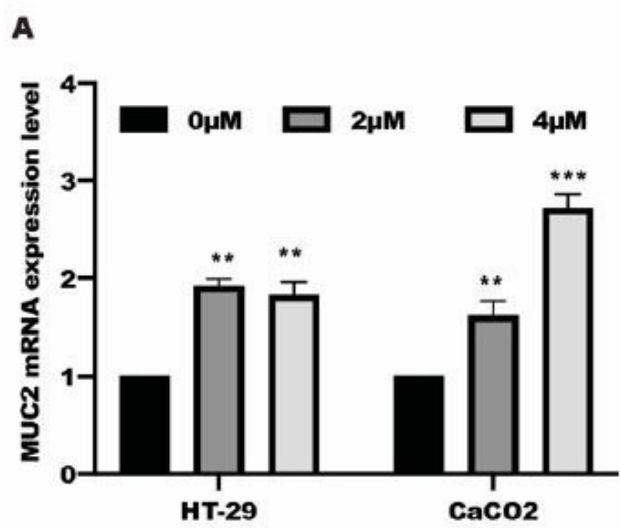

B
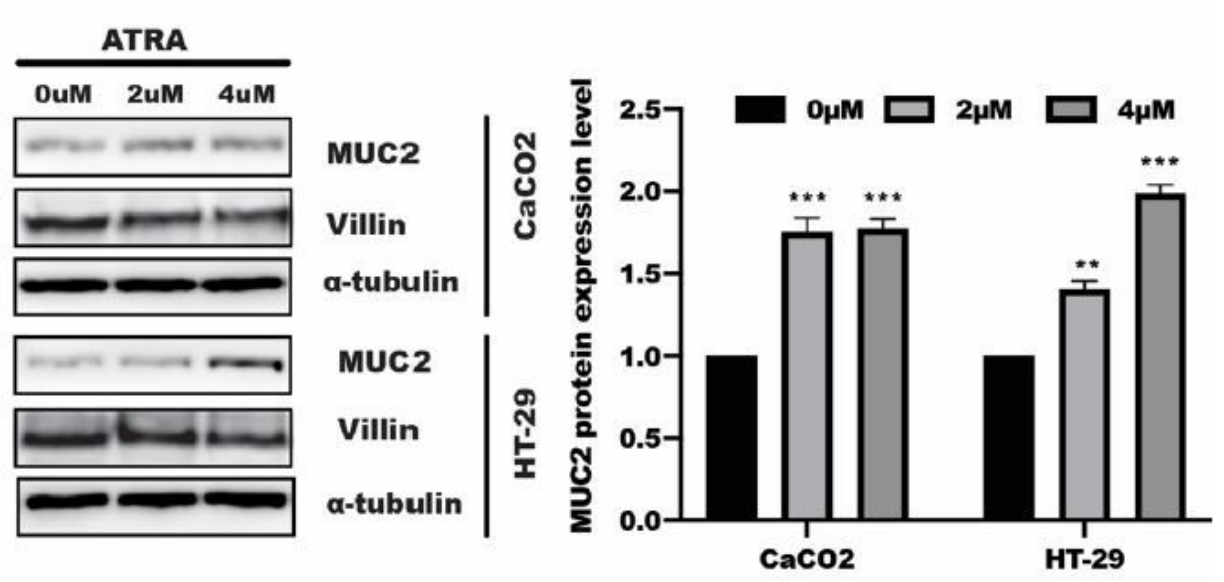

c

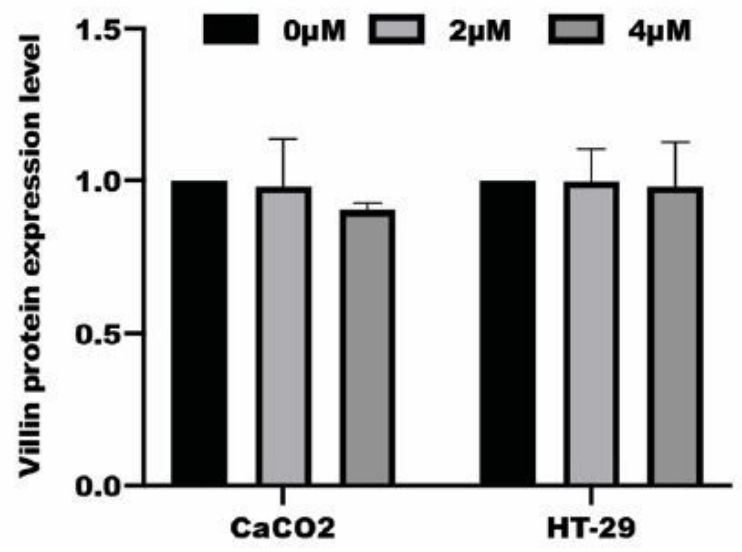

Figure 2

ATRA contributed MUC2 expression in IECs. (A) Real time-PCR analysis of MUC2 expression level in HT-29 and $\mathrm{CaCO} 2$ cells with or without ATRA stimulation for 48 hours, respectively. The statistical difference was performed by one-way ANOVA analysis, $n=3, * \star * P<0.001 ; * \star P<0.01$. Error bars indicated s.d. (B) left panel:HT-29 and CaCO2 cells were treated with or with ATRA for 48 hours, and the total protein were collected and analyzed indicated protein level. Right panel: the uantitation of MUC2 band in the western blotting were analyzed. (C) the intensity of villin were quantified in HT-29 and CaCO2 cells in indicated group and analyzed, respectively. 
Fig.3

A

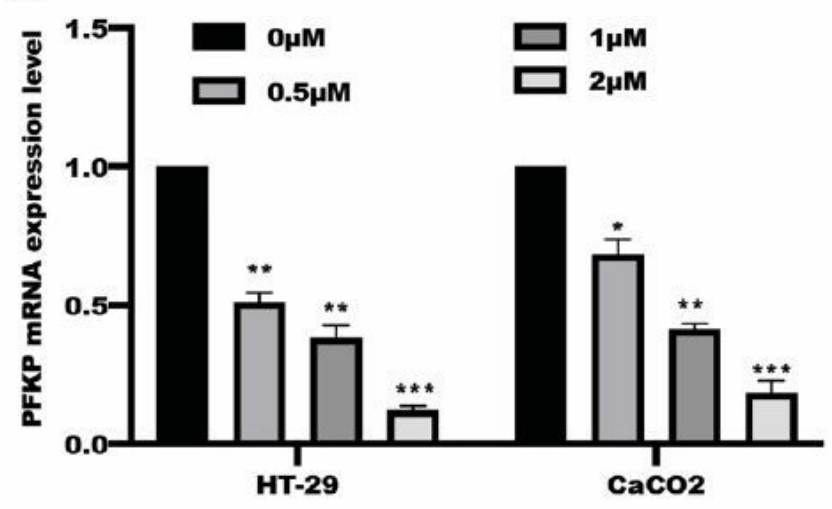

C

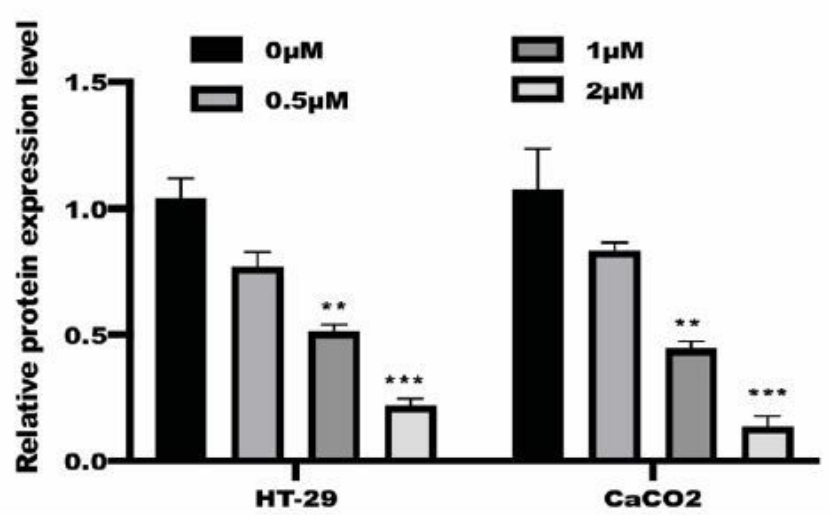

B

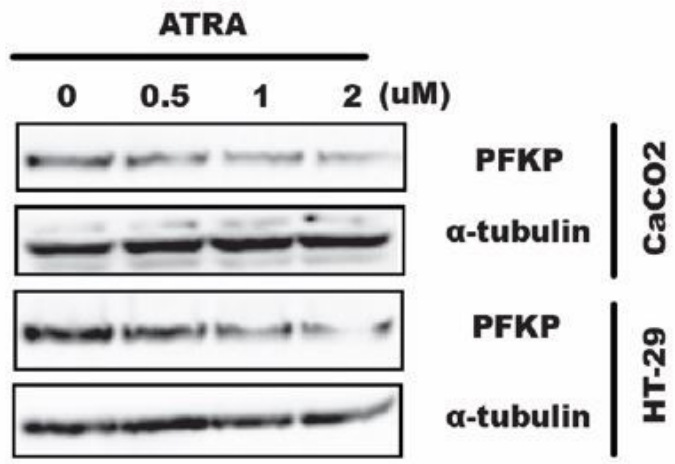

D

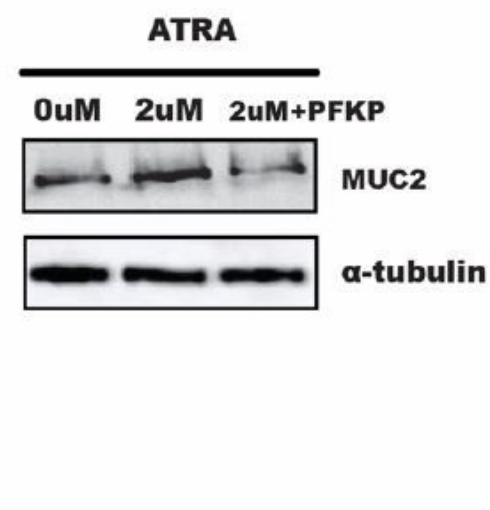

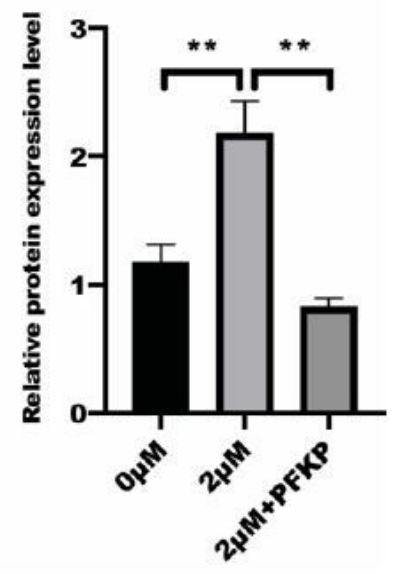

Figure 3

ATRA induced MUC2 expression in PFKP-dependent way. (A) real-time PCR analysis of PFKP in HT-29 and $\mathrm{CaCO} 2$ cells treated with indicated concentration of ATRA. (B) western blotting was performed to detect PFKP expression in HT-29 and $\mathrm{CaCO} 2$ cell in indicated group, and intensity band were quantified and analyzed the statistical difference (C). (D) western blotting was used to analyze MUC2 expression in indicated group in HT-29 cells, and the protein level of MUC2 were quantified and analyzed. 
Fig.4

A

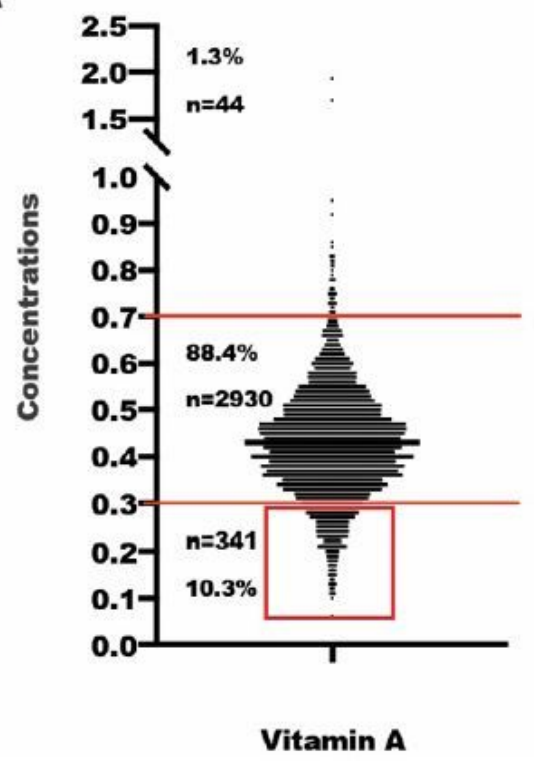

B

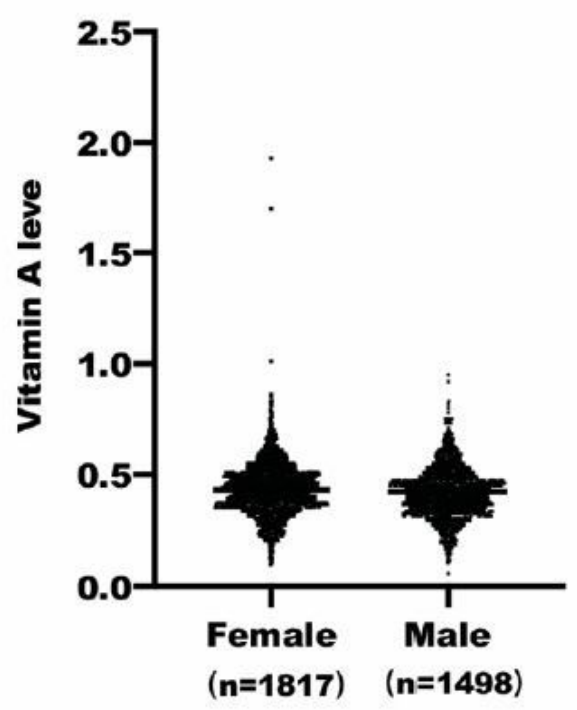

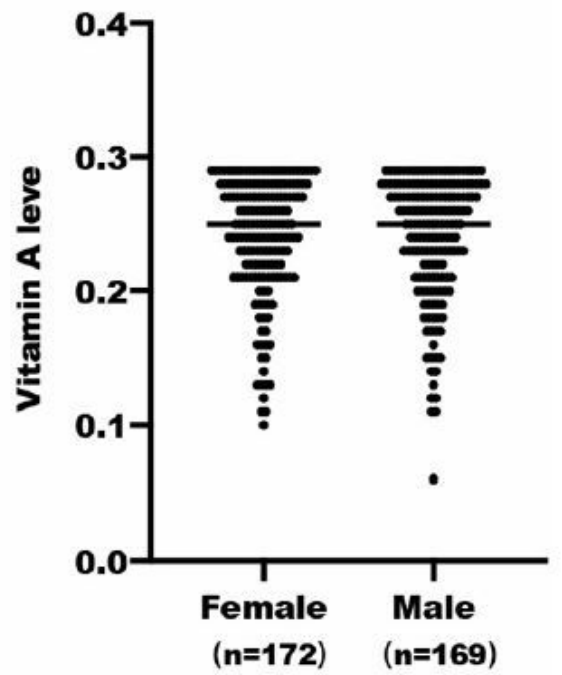

Figure 4

Clinical characteristic of Vitamin A. (A) the serum was collected to measure the level of vitamin A upon Written informed consent given. (B) statistical analysis was performed based on gender; (C) statistical difference were performed to analyze the population of low vitamin $A$ that marked red box in (A) up on gender.

\section{Supplementary Files}

This is a list of supplementary files associated with this preprint. Click to download.

- SupplementaryMaterials.xlsx 